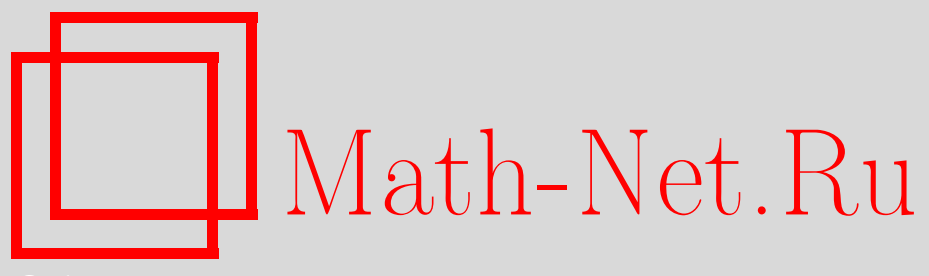

А. Б. Рамазанов, Об оценке кривизны порядкововыпуклого множества на целочисленной решетке и некоторые смежные вопросы, Матем. заметки, 2008, том 84, выпуск 1, 153-156

DOI: https://doi.org/10.4213/mzm4174

Использование Общероссийского математического портала Math-Net.Ru подразумевает, что вы прочитали и согласны с пользовательским соглашением http://www.mathnet.ru/rus/agreement

Параметры загрузки:

IP : 54.172 .240 .79

26 апреля 2023 г., $12: 27: 47$

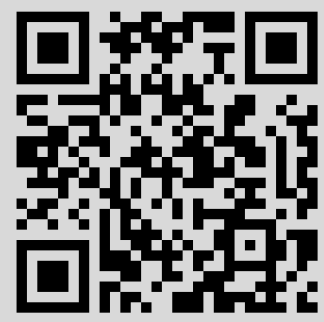




\section{Об оценке кривизны порядково-выпуклого множества на целочисленной решетке и некоторые смежные вопросы}

\section{А. Б. Рамазанов}

1. Введение. Известно (см., например, [1]-[3], др. ссылки см., [4]), что гарантированная оценка погрешности градиентного алгоритма решения некоторых задач дискретной оптимизации (ДО) может быть выражена через такую характеристику допустимой области, как кривизна. Кроме того, в некоторых случаях кривизна допустимой области позволяет эффективно охарактеризовать строение допустимого множества, а также сформулировать условие совпадения глобального и локального (градиентного) экстремумов в соответствующей задаче ДО [1]-[3]. Поэтому знание кривизны допустимого множества в некоторых случаях служит основой для оценки точности градиентного алгоритма, а также проверки оптимальности найденного градиентного решения в соответствующей задаче ДО. Не для всех допустимых множеств в задачах ДО можно найти точное значение кривизны, т.е. возникает необходимость оценки кривизны [1].

В работе получена оценка кривизны множества $P_{f}^{-}=\{x: f(x) \leqslant 0, f(0) \leqslant 0, x \in P\}$, где $-f(x)-\rho$-координатно-выпуклая функция, понятие которой введено в [5], $P$ - порядково-выпуклое множество в целочисленной решетке $\mathbb{Z}_{+}^{n}=\left(\mathbb{Z}_{+}^{n}, \leqslant\right), f(x)$ принимает целочисленные значение при любом $x \in P$. Эта оценка позволила определить близость глобального и градиентного решений в задачах ДО. Кроме того, предложено видоизмененное определение суперматроида и найдены условия совпадения глобального и градиентного экстремумов координатно-выпуклой функции в терминах кривизны указанного множества $P_{f}^{-}$. С помощью полученных результатов исследована устойчивость градиентного алгоритма относительно возмущения параметров допустимой области для одной задачи ДО.

2. Постановка задачи. Пусть $\mathbb{Z}_{+}^{n}-$ множество всех $n$-мерных неотрицательных целочисленных векторов. Если $0=(0, \ldots, 0) \in P \subseteq \mathbb{Z}_{+}^{n}, P$ конечное и из условия $x \leqslant y$, $x, y \in P$, следует включение $[x, y]=\left\{z: x \leqslant z \leqslant y, z \in \mathbb{Z}_{+}^{n}\right\} \subseteq P$, то множество $P$ называется конечным порядково-выпуклым с нулем [1]. Далее будем считать, что $P \subseteq \mathbb{Z}_{+}^{n}$ конечное порядково-выпуклое множество с нулем. Кривизной множества $P$ называется число $\theta(P)=\min \left\{l(P \cap[0, x]) / h(P \cap[0, x]): x \in \mathbb{Z}_{+}^{n}, x \neq 0\right\}$, где $l(Q)=\min \left\{h(x): x \in Q^{\max }\right\}$, $Q^{\max }$ - множество всех максимальных элементов частично упорядоченного множества $(Q, \leqslant), Q \subseteq P, h(Q)=\max \{h(x): x \in Q\}$ - высота множества $Q, h(x)=x_{1}+x_{2}+\cdots+x_{n}$, $x=\left(x_{1}, \ldots, x_{n}\right)$. Очевидно, что $0<\theta(P) \leqslant 1$. Если $\theta(P)=1$, то множество $P$ называется суперматроидом [1].

Функция $f: \mathbb{Z}_{+}^{n} \rightarrow \mathbb{R}$ (где $\mathbb{R}$ множество действительных чисел) называется координатно-вогнутой (координатно-выпуклой) [1], [5], если

$$
\Delta_{i j} f(x)=\Delta_{j} f\left(x+e^{i}\right)-\Delta_{j} f(x) \geqslant(\leqslant) 0 \quad \forall x \in \mathbb{Z}_{+}^{n}, \quad i, j \in I_{n}=\{1,2, \ldots, n\},
$$

где

$$
\Delta_{j} f(x)=f\left(x+e^{j}\right)-f(x), \quad e^{j}=\left(e_{1}^{j}, \ldots, e_{n}^{j}\right), \quad e_{j}^{j}=1, \quad e_{k}^{j}=0, \quad j \neq k, \quad j, k \in I_{n} .
$$

Как обычно, функцию $f: \mathbb{Z}_{+}^{n} \rightarrow \mathbb{R}$ будем называть неубывающей (не возрастающей), если $\Delta_{i} f(x) \geqslant 0\left(\Delta_{i} f(x) \leqslant 0\right) \quad \forall x \in \mathbb{Z}_{+}^{n}, i \in I_{n}$.

Рассмотрим задачу А дискретной оптимизации

$$
\max \{F(x): x \in P\},
$$

(C) А. Б. РАмазанов, 2008 
где $F(x)$ - неубывающая координатно-выпуклая функция.

Пусть $x^{*}$ - оптимальное решение задачи $\mathrm{A}$, а $x^{g}$ - градиентное решение задачи $\mathrm{A}$, т.е. точка, полученная с помощью градиентного алгоритма покоординатного подъема (см., например, [1], [5]-[7]). Под гарантированной оценкой погрешности градиентного алгоритма решения задачи А понимают такое число $\varepsilon \geqslant 0$, что

$$
\frac{F\left(x^{*}\right)-F\left(x^{g}\right)}{F\left(x^{*}\right)-F(0)} \leqslant \varepsilon .
$$

Известно (см., теорему 11.3 [1]), что гарантированная оценка погрешности градиентного алгоритма решения задачи А равна $1 /(\theta(P)+1)$. Если в задаче А $F(x)$ - сепарабельная неубывающая функция, то верно неравенство (см., теорему $11.4[1]) F\left(x^{g}\right) \geqslant \theta(P) F\left(x^{*}\right)+$ $(1-\theta(P)) F(0)$.

Аналогичные результаты для случая $\rho$-координатно-выпуклой целевой функции содержатся в [7]. Отметим, что первоначально результат подобного рода был получен в [2] для случая линейной целевой функции.

3. Оценка кривизны. Пусть $\rho=\left(\rho_{1}, \ldots, \rho_{n}\right) \in \mathbb{R}_{+}^{n}$, где $\mathbb{R}_{+}^{n}-$ множество $n$-мерных неотрицательных действительных векторов. Координатно-вогнутую (координатно-выпуклую) функцию $f(x)$ будем называть $\rho$-координатно-вогнутой ( $\rho$-координатно-выпуклой) [5], [7], если

$$
\Delta_{i i} f(x)-\rho_{i} \geqslant 0 \quad\left(\Delta_{i i} f(x)+\rho_{i} \leqslant 0\right) \quad \forall x \in \mathbb{Z}_{+}^{n}, \quad i \in I_{n} .
$$

Множество всех $\rho$-координатно-вогнутых ( $\rho$-координатно-выпуклых) функций будем обозначать через $\operatorname{Re}_{\rho}^{0}\left(\mathbb{Z}_{+}^{n}\right)=\operatorname{Re}_{\rho}^{0}\left(\operatorname{Re}_{\rho}=\operatorname{Re}_{\rho}\left(\mathbb{Z}_{+}^{n}\right)\right)$.

Теорема 1. Функиия $f(x)$ принадлежит классу $\operatorname{Re}_{\rho}^{0}\left(\mathbb{Z}_{+}^{n}\right)$ тогда и только тогда, когда она представима в виде

$f(x)=g(x)+\varphi(x)$,

где $g(x) \in \operatorname{Re}_{0}^{0}\left(\mathbb{Z}_{+}^{n}\right), \varphi(x)=\left(\rho_{1} x_{1}^{2}+\cdots+\rho_{n} x_{n}^{2}\right) / 2$.

Доказательство теоремы 1 аналогично доказательству теоремы 1 [5].

Теорема 2. Если неубывающая функиия $f(x) \in \operatorname{Re}_{\rho}^{0}\left(\mathbb{Z}_{+}^{n}\right)$, то множество $P_{f}^{-}$является порядково-выпуклым.

Доказательство теоремы вытекает из теоремы 6 [5] с учетом $-f(x) \in \operatorname{Re}_{\rho}\left(\mathbb{Z}_{+}^{n}\right)$ и $\Delta_{i} f(z) \geqslant 0 \forall i \in I_{n} \quad \forall z \in P$ (так как функция $f(x)$ неубывающая).

Tеорема 3. Пусть $f_{k}(x) \in \operatorname{Re}_{\rho^{k}}^{0}\left(\mathbb{Z}_{+}^{n}\right)$ - неубывающие функиии для $\forall k \in I_{m}, \rho^{k}=$ $\left(\rho_{1}^{k}, \rho_{2}^{k}, \ldots, \rho_{n}^{k}\right) \in \mathbb{R}_{+}^{n}, k \in I_{m} . E c л u f(x)=\max _{k}\left\{f_{k}(x): k \in I_{m}\right\}, \operatorname{mo} \theta\left(\bigcap_{k=1}^{m} P_{f_{k}}^{-}\right)=\theta\left(P_{f}^{-}\right)$.

Доказательство. Пусть $x \in P_{f_{1}}^{-} \cap \cdots \cap P_{f_{m}}^{-}$. Тогда $x \in P_{f_{k}}^{-} \forall k \in I_{m}$, т.е. $x \in P_{f}^{-}$. Аналогично, пусть $x \in P_{f}^{-}$. Тогда $0 \geqslant f(x) \geqslant f_{k}(x) \forall k \in I_{m}$. Поэтому $x \in \bigcap_{k=1}^{m} P_{f_{k}}^{-}$, т.е. справедливо утверждение теоремы 3.

В дальнейшем будем пользоваться следующими обозначениями: $\Omega^{*}(\rho, h)=\Omega^{0}(\rho, h)$, если $\Omega^{0}(\rho, h)>1$, и $\Omega^{*}(\rho, h)=1 / \Omega^{0}(\rho, h)$, если $0<\Omega^{0}(\rho, h) \leqslant 1$, где $\Omega^{0}=\Omega^{0}(\rho, h)=$ $(h+1 / 2) \Omega(\rho), h=h(P), \Omega(\rho)=\rho_{1}+\cdots+\rho_{n}$.

Теорема 4. Пусть $f(x) \in \operatorname{Re}_{\rho}^{0}\left(\mathbb{Z}_{+}^{n}\right)$ - неубывающая функиия, $\Omega(\rho)>0$, и пусть $g(x) \in$ $\operatorname{Re}_{0}^{0}\left(\mathbb{Z}_{+}^{n}\right)$ - невозрастающал функция. Тогда $\theta\left(P_{f}^{-}\right) \geqslant 1 / \Omega^{*}(\rho, h)$.

ДокАзАтельство. Так как $f(x) \in \operatorname{Re}_{\rho}^{0}\left(\mathbb{Z}_{+}^{n}\right)$, то на основании теоремы 1 функция $f(x)$ представима в виде (1) и в силу теоремы 2 множество $P_{f}^{-}$является порядково-выпуклым. Легко видеть, что $\Delta_{i} f(x) \leqslant \Omega^{0}(\rho, h) \forall x \in P \forall i \in I_{n}$.

Поэтому функция $\eta(x)=\Omega^{*}(\rho, h) h(x)-f(x)$ - неубывающая на множестве $P$. Далее доказательство теоремы 4 повторяет схему доказательства теоремы 5.2 [1]. 
СлЕДСТВиЕ 1. В условиях теоремъ 4 , если $\Omega^{0}(\rho, h)=1$, то множество $P_{f}^{-}$является суперматроидом.

Принимая во внимание следствие 1, можно переформулировать теорему 6.3 [1], которая является, по существу, одним из определений суперматроида, следующим образом.

СледСтвиЕ 2. Порядково-выпуклое множество $S \subseteq \mathbb{Z}_{+}^{n}$ является суперматроидом тогда и толъко тогда, когда существует такой вектор $\rho=\left(\rho_{1}, \ldots, \rho_{n}\right) \in \mathbb{R}_{+}^{n}$, ито $S$ совпадает с множеством $P_{f}^{-}$некоторой функиии $f(x) \in \operatorname{Re}_{\rho}^{0}\left(\mathbb{Z}_{+}^{n}\right)$.

Пусть $A=\left(a_{i j}\right)_{n \times n} \in \mathbb{R}_{+}^{n \times n}, a_{i j}=a_{j i} \forall(i, j) \in I_{n} \times I_{n}, x=\left(x_{1}, \ldots, x_{n}\right) \in \mathbb{Z}_{+}^{n}, b \in \mathbb{R}_{+}^{1}$, $b>0,(A x, x)$ - скалярное произведение векторов $A x$ и $x$. В пространстве $\mathbb{R}_{+}^{n \times n}$ зададим чебышевскую норму [8; гл. 6 , с. 175], т.е. под нормой матрицы $A=\left(a_{i j}\right)_{n \times n} \in \mathbb{R}_{+}^{n \times n}$ будем понимать число $\|A\|=\max \left\{a_{i j}:(i, j) \in I_{n} \times I_{n}\right\}$.

Теорема 5. Пусть $\psi(x)=(A x, x) / 2-b-$ неубывающая функция на множестве $P \subseteq \mathbb{Z}_{+}^{n},\|A\|>0$. Тогда имеем $\theta\left(P_{\psi}^{-}\right) \geqslant M(\|A\|, h)$, где $M(\|A\|, h)=(h+1 / 2)\|A\|$, если $(h+1 / 2)\|A\|<1, u M(\|A\|, h)=2 /(2 h+1)\|A\|$, еслu $(h+1 / 2)\|A\| \geqslant 1, h=h(P)$.

Доказательство теоремы повторяет схему доказательства теорем 5.2 [1] и 4.

СЛЕДСТВИЕ 3. В условиях теоремы 5 , если $M(\|A\|, h)=1$, то множество $P_{\psi}^{-}$является суперматроидом.

4. Гарантированные оценки. Применим полученные результаты для нахождения гарантированной оценки погрешности градиентного алгоритма в задачах максимизации координатно-выпуклой функции на множестве $P_{f}^{-}$.

Рассмотрим следующие задачи. $\mathbb{R}_{+}^{n}$.

ЗАДАчА В. $\max \left\{F(x): x \in P_{f}^{-}\right\}$, әде $F(x) \in \operatorname{Re}_{0}\left(\mathbb{Z}_{+}^{n}\right), f(x) \in \operatorname{Re}_{\rho}^{0}\left(\mathbb{Z}_{+}^{n}\right), \rho=\left(\rho_{1}, \ldots, \rho_{n}\right) \in$

ЗАдАчА С. $\max \left\{F(x)=\sum_{i=1}^{n} F_{i}\left(x_{i}\right): x \in P_{f}^{-}\right\}$, әде $F_{i}\left(x_{i}\right) \in \operatorname{Re}_{0}\left(\mathbb{Z}_{+}^{1}\right), i \in I_{n}-$ неубъвающая функиия.

Из теорем 4, 5 и из упомянутой в п. 2 теорем 11.3 [1], 11.4 [1] следуют.

Теорема 6. Пусть в задаче В $F(x)$-неубывающая функиия на множестве $P \subseteq \mathbb{Z}_{+}^{n}$. Тогда гарантированная ощенка погрешности градиентного алгоритма решения задачи В выражается формулой $\varepsilon=\Omega^{0}(\rho, h) /\left(1+\Omega^{0}(\rho, h)\right)$, если $\Omega^{0}(\rho, h)>1, u \varepsilon=1 /\left(1+\Omega^{0}(\rho, h)\right)$, если $0<\Omega^{0}(\rho, h) \leqslant 1$.

ТЕорема 7. В условиях теоремы 5 гарантированная оценка погрешности градиентного алгоритма решения задачи максимизации неубывающей функции $F(x) \in \operatorname{Re}_{0}\left(\mathbb{Z}_{+}^{n}\right)$ на множестве $P_{\psi}^{-}$выражается бормулой $\varepsilon=1 /(1+M(\|A\|, h))$.

Теорема 8. Градиентное решение $x^{g}$ задачи С удовлетворяет неравенствам

$$
F\left(x^{g}\right) \geqslant \begin{cases}\frac{1}{\Omega^{0}(\rho, h)} F\left(x^{*}\right)+\left(1-\frac{1}{\Omega^{0}(\rho, h)}\right) F(0), & \text { если } \Omega^{0}(\rho, h)>1, \\ \Omega^{0}(\rho, h) F\left(x^{*}\right)+\left(1-\Omega^{0}(\rho, h)\right) F(0), & \text { если } \Omega^{0}(\rho, h) \leqslant 1 .\end{cases}
$$

Отсюда в случае, когда $\Omega^{0}(\rho, h)=1$, получаем хорошо известный (см., например, [1], [6]) результат о совпадении глобального и градиентного экстремумов задачи максимизации сепарабельной координатно-выпуклой функции на суперматроиде.

Определим множество возмущающих $B^{\delta}$ матриц порядка $n \times n$ следующим образом [9]: $B^{\delta}=\left\{B \in \mathbb{R}_{+}^{n \times n}: 0<\|B\|<\delta, \delta \in \mathbb{R}_{+}^{1}\right\}$.

Рассмотрим следующую задачу D ДО: $\max \left\{F(x): x \in P_{\psi}^{-}\right\}$. 
Под возмущением задачи $\mathrm{D}$ по матрице $A=\left(a_{i j}\right) \in \mathbb{Z}_{+}^{n \times n}$ будем понимать задачи $\mathrm{D}^{\delta}$ : $\max \left\{F(x): x \in P_{\psi}^{\delta}\right\}$, где $P_{\psi}^{\delta}=\left\{x:((A+B) x, x) / 2-b \leqslant 0, B \in B^{\delta}, x \in P\right\}$.

Пусть $\varepsilon\left(\varepsilon^{\delta}\right)$ - гарантированная оценка погрешности градиентного алгоритма некоторой исходной (возмущенной) задачи ДО. Как обычно, градиентный алгоритм будем называть устойчивым, если $\varepsilon^{\delta}<\varepsilon K(\delta)$, где $K(\delta) \rightarrow 1$ при $\delta \rightarrow 0$.

Теорема 9. Пусть $\varepsilon$ и $\varepsilon^{\delta}$ - соответственно гарантированные оценки погрешности градиентного алгоритма для задачи $D$ и $D^{\delta}$. Тогда $\varepsilon^{\delta}<\varepsilon(1+\delta /\|A\|)$.

Доказательство теоремы вытекает из теорем 5,7 с учетом соотношений

$$
\begin{aligned}
\varepsilon^{\delta} & =\frac{(2 h+1)\|A+B\|}{2+(2 h+1)\|A+B\|}<\frac{(2 h+1)\|A\|+(2 h+1)\|B\|}{2+(2 h+1)\|A\|} \\
& <\varepsilon+\frac{(2 h+1) \delta}{2+(2 h+1)\|A\|}=\varepsilon\left(1+\frac{\delta}{\|A\|}\right) .
\end{aligned}
$$

\section{СПИСОК ЦИТИРОВАННОЙ ЛИТЕРАТУРЫ}

[1] М. М. Ковалев, Матроиды в дискретной оптимизации, Изд-во Университетское, Минск, 1987. [2] B. Korte, D. Hausmann, Ann. Discrete Math., 2 (1978), 65-74. [3] М. М. Ковалев, П.Б. Миланов, ЖКВМ и МФ, 24:5 (1984), 786-790. [4] М. М. Ковалев, Вестн. Белорусского гос. ун-та. Сер. 1. Физ. Матем. Информ., 1:3 (2001), 44-51. [5] В.А. Емеличев, М. М. Ковалев, А.Б. Рамазанов, Дискрет. матем., 2:2 (1990), 127-137. [6] Н. И. Глебов, Управляемые системы, вып. 17, ИМ СО АН СССР, Новосибирск, 1978, 52-59. [7] А. Б. Рамазанов, Дискретн. анализ и исслед. опер., серия 1, 12:4 (2005), 60-80. [8] П. Ланкастер, Теория матрии, Наука, М., 1982. [9] В. А. Емеличев, В. Н. Кричко, Дискрет. матем., 16, 2004.

\section{А. Б. Рамазанов}

Поступило

Бакинский государственный университет

18.12.2007

E-mail: rab-unibak@rambler.ru 